\title{
Potential Use of Natural Ingredients as Alternative Chemical Wash
}

\author{
Naresh Shahi ${ }^{1}$ and Byungjin Min ${ }^{2 *}$ \\ ${ }^{1}$ Department of Integrative Biosciences, Tuskegee University, USA \\ ${ }^{2}$ Department of Food and Nutritional Sciences, Tuskegee University, USA
}

*Corresponding author: Byungin Min, Department of Food and Nutritional Sciences, Tuskegee University, Tuskegee, AL, USA.

Received Date: November 20, 2019

Published Date: January 13, 2020

\begin{abstract}
Proper washing treatment of fresh produce plays a critical role to reduce or minimize microbial contaminations for safe consumption. This study investigated antimicrobial activities of plant-based ingredients as an alternative wash to substitute chemical wash. From the preliminary test, five solutions: 1) $0.5 \%$ white distilled vinegar (DV); 2) $25 \%$ crude lemon juice (LJ); 3) DV + 0.1\% origanum oil (DVO); 4) LJ+ 0.1\% origanum oil (LJO) and 5) DV+ LJ $+0.1 \%$ origanum oil (DVLJO) and sterile water (control) were selected. To evaluate antimicrobial activities against foodborne pathogens both L. monocytogenes and S. Typhimurium zone of inhibition (ZOI), minimum inhibition concentration (MIC) and minimum bactericidal concentration (MBC) were measured. In addition, model study in an aqueous solution was designed to determine effective washing time against tested microorganisms, and it was performed at 2, 5, 15, 20 and 25 min. MICs and MBCs of natural ingredients against L. monocytogenes and S. Typhimurium were 0.03 to $0.78 \%$ and 0.06 to $1.56 \%$, respectively, but higher concentration was required for lemon juice extract (12.5\%). The combined treatment DVLJO exhibited the least MIC $(0.03 \%)$ as well as MBC $(0.06 \%)$ against L. monocytogenes. The results indicated that combination of wash solutions reduced bacterial populations by $\sim 3$ to $5 \log \mathrm{CFU} / \mathrm{mL}$ at $25 \mathrm{~min}$ of agitation. However, there were no significant differences in bacterial reductions by washing time between 5 and $25 \mathrm{~min}(\mathrm{P}>0.05)$. Based on the results, it is suggested that combinations of vinegar, lemon juice and essential oil might be suitable as an alternative antimicrobial wash solution for raw or minimally processed foods. It is thought that formulated wash solutions with natural ingredients are readily available and easy to use at the house-hold level. However, further study is recommended to validate and specify the effectiveness of wash solutions in a real food system.
\end{abstract}

Keywords: Vinegar; Essential oil; Natural antimicrobials

Abbreviations: ATCC: American Type Culture Collection; CFU: Colony-Forming Unit; DV: White Distilled Vinegar; DVO: DV + Origanum Oil; LJ: Crude Lemon Juice; DVLJO: DV+ LJ + Origanum Oil; EO: Essential Oils; LJO: LJ+ Origanum Oil; MBC: Minimum Bactericidal Concentration; MIC: Minimum Inhibitory Concentration; OD: Optical Density; TSA: Tryptic Soy Agar; ZOI: Zone of Inhibition

\section{Highlights}

$>$ Natural ingredients as potential substantially reduced microbial growth.

$>$ A combination of the natural solution was more effective than a single use of antimicrobial.

> Natural wash could be used to decontaminate fresh produce.

\section{Introduction}

Various intervention technologies such as physical, chemical, and biological treatments have been employed to control foodborne pathogens $[1,2]$. Compared to generic chemical methods, plantbased decontamination methods gaining popularity because it is generally considered as safe in a food matrix [3,4]. Also, plant-based antimicrobials are readily available and easy-to-use as compared to physical treatments [5]. Over the past few years, interests of using essential oils (EOs) and organic acids have been increased due to their significant antimicrobial activities against a broad spectrum of foodborne pathogenic bacteria [6-9].

Organic acids such as citric acid, acetic acid and lactic acid are impregnated into foods to enhance food safety and meat quality in particular [10]. Organic acids are being used in the food industry as preservative due to their acidic properties since concentrations of hydrogen ions impact the growth and survival of microorganisms in foods $[2,5]$. Several types of conventional vinegars are available on the market, and these are rice vinegar, distilled vinegar, balsamic vinegar, wine vinegar, black vinegar, bamboo vinegar, etc. In general, 
vinegars from fermented carbohydrate contains 3.75 to $5 \%$ of acetic acid as an active ingredient [9]. Vinegar can inhibit a wide range of bacteria and used as an active decontamination agent, especially for fresh produce [11,12]. Likewise, EOs are also considered as natural antimicrobials which are colorless, complex, and comprise of volatile aromatic compounds present in plants [13]. The antimicrobial activities of EOs are due to their hydrophobic nature, in which it disrupts the cell membrane and cell wall of the bacteria [7]. Correspondingly, lemon is considered one of major citrus fruit along with oranges and mandarins. The essential compounds in lemon juice are water, citric acid, and carboxylic acid. It is reported that raw or partially cooked beef meats marinated with lemon juice along with heat treatment reduced the risk of foodborne pathogens [10].

Microbial food safety of raw or minimally processed fresh produce is a challenge in the food supply chain because it increases the risk of illness and causes significant reduction in shelf-life [14]. Current chemical treatment such as chlorine, nitric oxide, peroxyacetic acid, hydrogen peroxide, quaternary ammonia, and ozone are effective in reducing the microbial load in wash water. However, they have limited efficacy for the bacterial inactivation on fresh produce, by causing food oxidation, and possible risk of releasing carcinogenic by- products [15-18]. On the other hand, plant-based antimicrobials are alternative as they are often devoid of the many side effects as compared to synthetic chemicals. A potential use of plant-based ingredients with optimized formulations in washing treatment might be an alternative way to reduce or replace harmful chemicals use. Therefore, this study was designed to evaluate antimicrobial activities of natural ingredients such as vinegar, lemon juice and essential oil, and to compare antibacterial effectiveness of cocktail combinations against foodborne pathogens.

\section{Materials and Methods}

\section{Collection of natural antimicrobials}

Fresh lemons and different brands of conventional vinegar: white distilled vinegar, seasoned rice vinegar, balsamic vinegar, apple cider vinegar, and red wine vinegar were purchased from local grocery stores in Alabama, USA. Lactic acid ( $-(+)-)$ solution (88-92\%), Origanum oil (100\%) were purchased from SigmaAldrich Company (St. Louis, USA) and stored at $4{ }^{\circ} \mathrm{C} \pm 0.5$ until use.

\section{Screening of natural antimicrobials}

Selected vinegars are diluted with sterile distilled water to prepare $4 \%$ of stock solution. For lemon juice extract (LJ), fresh lemons were washed and cut with a sterile knife. The household juicer was used to obtain fresh LJ. Extracted crude lemon juice was filtered using filter paper (Whatman ${ }^{\circledR}$ No. 4). Filtered LJ was dissolved into sterile water $(1: 1 \mathrm{~V} / \mathrm{V})$ to make $50 \%$ lemon juice.

\section{Culture preparation}

Antimicrobial activities of natural chemicals were tested against L. monocytogenes (ATCC 13912) and Salmonella enteric
Typhimurium (ATCC 51812). Test bacteria were obtained from the stock culture collection of the Food Safety Laboratory, Department of Food and Nutritional Sciences, Tuskegee University. Stock cultures were stored in Tryptic Soy Broth (Fulka analytical 22091, Sigma-Aldrich) at below $4{ }^{\circ} \mathrm{C}$. Cultures were streaked onto TSA plates and incubated at $37{ }^{\circ} \mathrm{C}$ for $18 \pm 1 \mathrm{~h}$. Subsequently, a colony of L. monocytogenes and Salmonella Typhimurium was aseptically inoculated into $5 \mathrm{~mL}$ TSA broth and incubated at $37^{\circ} \mathrm{C}$ for $18 \pm 1$ h. To achieve a viable cell population of 8-9 Log CFU/mL, $100 \mu \mathrm{L}$ of bacterial suspension was transfer into $5 \mathrm{~mL}$ TSA broth and incubated at $37{ }^{\circ} \mathrm{C} 18 \pm 1 \mathrm{~h}$. Bacterial culture was harvested by centrifugation at 5,000 rpm (Brofuge 22R, Heraeus Instruments, Inc., USA) for $5 \mathrm{~min}$ at $4{ }^{\circ} \mathrm{C}$. The supernatant was carefully discarded, and the pellet was resuspended in sterile peptone water $(0.1 \%)$ and thoroughly mixed by vortexing. Centrifugation and experiments were performed at least twice throughout the study. The suspension of washed cells was serially diluted (1:10) up to $6 \log _{10} \mathrm{CFU} / \mathrm{mL}$ in sterile peptone water $(0.1 \%)$ to obtain an appropriate cell concentration. Defined numbers of inocula were determined by colony counting from the $18 \mathrm{~h}$ culture grown on TSA from each diluent. Optical density (at 600nm) of bacterial culture solution was measured using a spectrophotometer (Genesys $10 \mathrm{UV}$, Thermo Electron Corporation) and adjusted to 0.87 , approximately $10^{6-7} \mathrm{CFU} / \mathrm{mL}$ of bacterial populations.

\section{Determination of antimicrobial activities using agar diffusion assay}

The agar diffusion assay was performed to screen and evaluate the antimicrobial activities of natural wash solutions. Tryptic Soy Agar (soft) was prepared in Petri dishes (90 mm diameter) with approximately $10^{6-7} \mathrm{CFU} / \mathrm{mL}$ of bacterial concentration. Sterile cork borer was used to prepare wells in the agar plates. In each well, $40 \mu \mathrm{L}$ of treatment was carefully dispensed and incubated at $37^{\circ} \mathrm{C}$ for $24 \mathrm{~h}$. Recorded zone of inhibition (ZOI) from the agar diffusion assay, measurements were taken from the clear zone around the well, including the well diameter. The test was performed in duplicate for confirmation.

\section{Minimum inhibitory concentration (MIC) and Minimum bactericidal concentration (MBC)}

The MICs of treatments were determined by standard broth microdilution. A 96-well microplate was used to determine the MICs. TSA broth was used as diluents and growth medium for the bacteria. Initially, $150 \mu \mathrm{L}$ of TSA broth was aseptically dispensed into micro-wells, and an equal volume of the treatment was added, making a total of $300 \mu \mathrm{L}$ as final volume. Subsequently, the mixture of TSA broth and treatments were properly mixed, and two-fold serial micro-dilutions were performed. Standardized inocula of 6-7 $\mathrm{Log} \mathrm{CFU} / \mathrm{mL}$ in $10 \mu \mathrm{L}$ was added into each well except positive control (without treatments) and incubated at 37 ${ }^{\circ} \mathrm{C}$ for $24 \mathrm{~h}$. The results were confirmed through turbidity resulted from the bacterial growth. Turbidity in each well were measured after $24 \mathrm{~h}$. The MICs were recorded as the lowest concentration of treatment by no growth observation which means no turbidity 
is measured. The MBCs of natural ingredients were determined by standard broth microdilution. All the microwells containing organisms and treatment along with positive and negative controls were sub-cultured onto TSA plate. Then each plate was divided and labeled into ten equal compartments and inoculated with 10 $\mu \mathrm{L}$ of suspension from microplate to respective compartments and incubated at $37{ }^{\circ} \mathrm{C}$ for $24 \mathrm{~h}$. After incubation, each well was examined for bacterial growth and MBC. If bacterial growth is fully inhibited by natural solutions, it was recorded as MBC. The remained content of the 96 well plates used for MIC and MBC were further subjected to perform bacterial growth in the liquid medium. This test is performed by measuring the optical density (OD) of bacterial growth in each well using a Bio Teck plate reader (Bio Teck, USA) at $600 \mathrm{~nm}$.

\section{Preparation of antimicrobial natural wash solutions}

Five different natural antimicrobial wash solutions were selected. Wash solutions were prepared in $100 \mathrm{~mL}$ of cultural bottles using sterile water. Natural antimicrobials used for wash solutions were $0.5 \%$ white distilled vinegar (DV), $25 \%$ crude lemon juice extract (LJ), and combination of both DV and LJ in origanum oil includes; DVO (DV $+0.1 \%$ origanum oil), $\mathrm{LJO}$ ( $\mathrm{LJ}+0.1 \%$ origanum oil), DVLJO (DV + LJ + 0.1\% origanum oil), all the samples were in total Vol/Vol percentage. Wash solutions were prepared on the same day of the experiment and stored in the refrigerator at $4{ }^{\circ} \mathrm{C}$ \pm 0.5 until used. The $\mathrm{pH}$ of the wash solutions was measured by $\mathrm{pH}$ meter (Denver Instrument, Model 215, USA). The measurement was performed in triplicates at $23{ }^{\circ} \mathrm{C} \pm 2$.

\section{A model study in the natural wash solution}

In vivo study was performed to determine the antimicrobial activities of each wash solution (DV, LJ, DVO, LJO, and DVLJO) and sterile water (control). During the study, an inoculum of Salmonella spp. and L. monocytogenes were inoculated into wash solutions. The concentration of inoculum in the wash solution was 6-7 log CFU/mL. The wash solution contained test organisms continuously agitated in an automatic shaker in $100 \mathrm{rpm}$ (Gyratory- Shaker Model G2, New Brunswick Scientific Co., USA) at room temperature $\left(23^{\circ} \mathrm{C} \pm 2\right)$ for $25 \mathrm{~min}$. The solution contained test organism was withdrawn $(20 \mu \mathrm{L})$ at $2,5,10,15,20$, and 25 min intervals and dispensed onto respective compartments of TSA plates and incubated for $24 \mathrm{~h}$ to examine the growth of inoculated organisms. Simultaneously, 100 $\mu \mathrm{L}$ of the solution was dispensed onto another set of TSA plates and spread homogenously, incubated for $24 \mathrm{~h}$ to determine the exact number of colonies. The process was replicated for the confirmation of the results.

\section{Statistical analysis}

Throughout the study, all experiments with assays were carried out in triplicates. Analysis of variance among the treatments from collected data was performed by repeated measurement analysis of variances (RM- ANOVA) of the general model procedure using a statistical system SAS 9.3 (SAS Inst., Cary, N.C., U.S.A.). Mean comparisons were calculated using Fisher's Protected least significant difference. Plate count data for bacterial populations were converted to a logarithmic scale before statistical analysis. The level of significance was determined at $\mathrm{P}<0.05$.

\section{Results and Discussion}

\section{Determination of antimicrobial activities using agar diffusion assay}

As presented in Table 1, zone of inhibition (ZOI) by treatments against L. monocytogenes and $S$. Typhimurium were determined using the agar diffusion assay. It is noted that inhibition zone around agar well is formed by bactericidal or bacteriostatic activity, while the absence of ZOI is generally considered as no antimicrobial effect on tested organisms.

Table 1: Zone of inhibition (ZOI) of selected natural ingredients against $\mathrm{L}$. monocytogenes and S. Typhimurium.

\begin{tabular}{|c|c|c|c|}
\hline \multicolumn{2}{|c|}{} & \multicolumn{2}{|c|}{ ZOI (mm) } \\
\hline Treatment & Conc. (\%, V/V) & L. monocytogenes & S. Typhimurium \\
\hline DV & 0.5 & - & - \\
\hline O & 0.1 & - & - \\
\hline LJ & 25 & 13 & 12 \\
\hline DVO & $0.5 / 0.1$ & 12.5 & 12.5 \\
\hline LJO & $25 / 0.1$ & 17.5 & 14.5 \\
\hline DVLJO & $0.5 / 25 / 0.1$ & 11.25 & 12.5 \\
\hline
\end{tabular}

DV: $0.5 \%$ white distilled vinegar; O: $0.1 \%$ origanum oil, LJ: $25 \%$ crude lemon juice; DVO: $0.5 \%$ white distilled vinegar $+0.1 \%$ origanum oil; LJO: $25 \%$ crude lemon juice $+0.1 \%$ origanum oil; DVLJO: $0.5 \%$ white distilled vinegar $+25 \%$ crude lemon juice $+0.1 \%$ origanum oil.

(-) sign indicates no antimicrobial activities or $<8 \mathrm{~mm}$ ZOI.

Crude lemon juice, origanum oil, and their combinations showed inhibitory activities against foodborne pathogenic bacteria on both L. monocytogenes and S. Typhimurium (Table 1). A combination of natural ingredients DVO, LJO, and DVLJO resulted in a distinct and clear inhibition zone against $L$. monocytogenes and $S$. typhimurium. Among the combinations of wash solutions, treatment LJO exhibited the highest ZOI of 17.5 and $14.5 \mathrm{~mm}$ against L. monocytogenes and S. Typhimurium, respectively. Similarly, ZOI by treatment LJ was 13 and $12 \mathrm{~mm}$ against L. monocytogenes and S. Typhimurium, respectively. Our results showed that combined treatments with natural ingredients were more effective than single ingredient alone.

It is reported that the antimicrobial activities of available commercial EOs and oreganum were evaluated on the growth of various foodborne pathogenic microorganisms such as Vibrio spp., Bacillus subtilis, E. coli, S. aureus, and L. monocytogenes $[13,19]$. Bioactive compounds such as thymol, eugenol and carvacrol which is found in EOs from oreganum, savory, lemongrass, and cinnamon exhibited potent and broad-spectrum of antimicrobials activities. It is assumed that the mode of antimicrobial action of these compounds on microorganisms is due to the disruption of the cell membrane and inhibition of ATPase activities [20]. $L$. monocytogenes exposed to the EO showed the inhibition of glucose metabolic pathway and rupturing the cell wall [21]. 


\section{Minimum inhibitory concentration (MIC)/minimum bactericidal concentration (MBC)}

The antimicrobial activities of natural ingredients in a TSB broth were performed to determine the MIC and MBC of samples. Procedures for knowing the minimum concentration of antimicrobial agents is a critical to distinguish whether samples at the certain level are effectively inhibit microorganisms or not. The MIC results from the present study showed that stronger activities against test organisms were proportional to increase of concentration. As presented in Table 2, synergistic antimicrobial effects on MIC and MBC was found in the combination of vinegar, lemon juice, and origanum oil.

Table 2: MICs and MBCs of selected natural wash solutions.

\begin{tabular}{|c|c|c|}
\hline Treatment & $\begin{array}{c}\text { Organisms tested }\left(10^{6-7}\right. \\
\text { CFU } / \mathrm{mL})\end{array}$ & MIC (MBC) (\%) \\
\hline DV & L. monocytogenes & $0.50(0.50)$ \\
\hline & S. Typhimurium & $0.3(0.50)$ \\
\hline & L. monocytogenes & $6.25(6.25)$ \\
\hline LJ & S. Typhimurium & $12.50(12.50)$ \\
\hline & L. monocytogenes & $0.78(1.56)$ \\
\hline LJO & S. Typhimurium & $0.78(1.56)$ \\
\hline & L. monocytogenes & $0.06(0.12)$ \\
\hline DVO & S. Typhimurium & $0.12(0.25)$ \\
\hline & L. monocytogenes & $0.03(0.06)$ \\
\hline DVLJO & S. Typhimurium & $0.25(0.25)$ \\
\hline
\end{tabular}

Essential oils in the combination with distilled vinegar and lemon juice showed the least MIC and MBC values against both organisms tested. It is assumed that higher antimicrobial activities of essential oil, and combined treatments with other antimicrobial substances were attributed to the high content of phenolic derivatives such as carvacrol. A combination of EOs and acetic acid enhancing inhibitory effects against Staphylococcus aureus has been reported [22]. Correspondingly, antimicrobial susceptibility of natural ingredients was more sensitive to gram-positive bacteria than gram-negative bacteria [23]. In this study, MIC and MBC values of the DV was $0.5 \%$. However, it is reported that household vinegars at high concentrations ( 2.5 and $5 \%$ of acetic acid) were effective to inhibit L. monocytogenes, E. coli 0157:H7, and Salmonella Typhimurium [24]. Another study showed that the MICs of origanum oil against gram-positive bacteria were 0.15 to $0.50 \mu \mathrm{L} /$ $\mathrm{mL}$ [25]. Selected natural wash solutions in our study exhibited substantial inhibitory activities in the liquid medium $(0.01 \%)$ than in the solid medium (0.5\%). Similar results were reported with many EOs in a liquid and solid medium, in which relatively higher concentration (5\%) was effective against test organisms in the solid medium [26].

Factors that influence microbial growths by organic acids depend on several parameters including $\mathrm{pH}$, chain length, and the ratio of undissociated ions [27]. It is assumed that weak organic acids can easily penetrate the plasma membrane and destroy the cell's genetic materials because weak acid can diffuse through the bacterial cell wall [12]. In this study, the $\mathrm{pH}$ of solutions with natural ingredients was ranged from 2.21 to 2.73 (Figure 1), indicating that majority of acidic solutions might be in an undissociated forms.

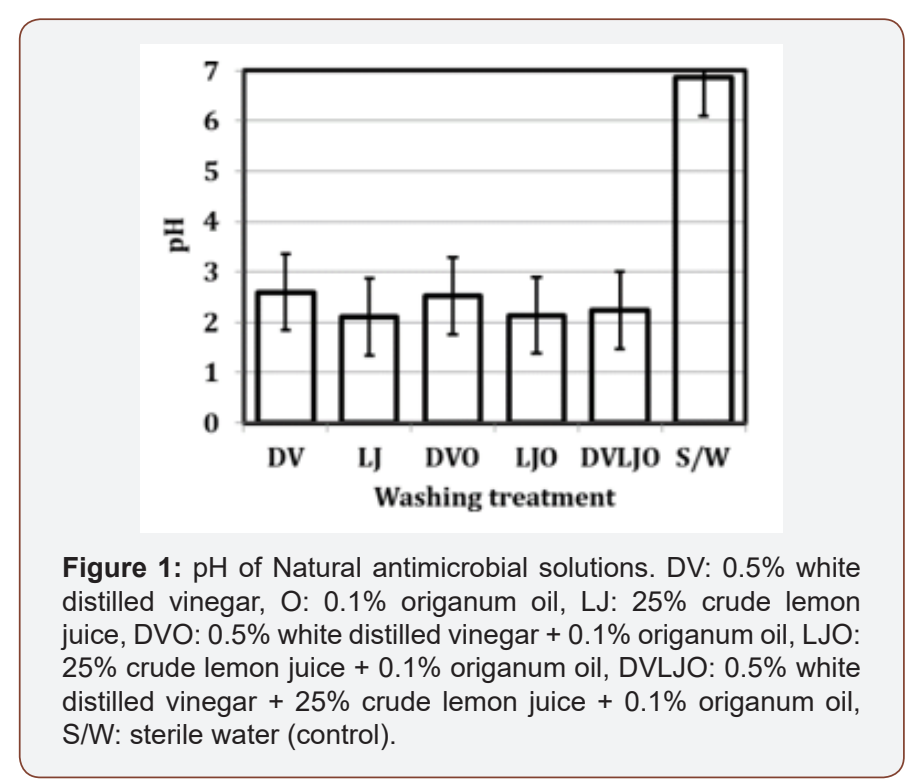

\section{Determination of optical density of tested organisms}

Antimicrobial activities of natural solutions were tested using twofold micro-dilution (concentrations ranged from 0.015 to $2.0 \%$ ) against $L$. monocytogenes and $S$. Typhimurium in a TSB broth. There was a significant difference $(\mathrm{p}<0.05)$ in inhibitory concentrations among DV, LJ, DVO, and DVLJO against L. monocytogenes. Contrary to $L$. monocytogenes, OD values for $S$. Typhimurium were in the higher concentration range of treatments ( 0.12 to $0.5 \%$ ). However, OD of solutions DV, LJO, and DVLJO were significantly different $(p<0.05)$. The antimicrobial activities of the natural solutions at the lower concentration $(0.015 \%)$ were more effective against grampositive organism than gram-negative organism (Table $3 \& 4$ ). The OD results demonstrate that inhibitory activities of antimicrobial solutions used in this study exhibited consistent results obtained from MIC and MBC. Interestingly, it is observed that there was an unusual high OD value in lemon juice treatment at $1 \%$. It might be due to increase of turbidity by lemon juice itself at relatively high concentration. Thus, increased OD value resulted from lemon juice at high concentration does not necessarily mean that it can be considered increased growth of microorganisms.

\section{A model study in an aqueous solution}

A model study was carried out to determine potential use of plant-based ingredients in a natural wash solution for fresh or minimally processed foods. As shown in Figure 2, reduced bacterial populations of L. monocytogenes and $S$. Typhimurium with different solutions were observed while there was no effect on bacterial reductions by sterile water as control. The bacterial reductions of L. monocytogenes by treatment DVO was higher in which approximately $4 \log \mathrm{CFU} / \mathrm{mL}$ followed by LJO with approximately 3 $\log \mathrm{CFU} / \mathrm{mL}$ after $5 \mathrm{~min}$. However, the highest microbial reductions of $S$. Typhimurium were obtained by DVLJO and LJO which was approximately $3 \log \mathrm{CFU} / \mathrm{mL}$ followed by DVO with approximately $2 \log \mathrm{CFU} / \mathrm{mL}$ after $5 \mathrm{~min}$. Results from the model study revealed 
that wash solutions with combined treatments DVO, LJO, and DVLJO were the most effective to suppress microbial growths of tested organisms. It was observed that natural solutions DV and LJ inhibited microorganisms after $20 \mathrm{~min}$, but antimicrobial activities by log reductions were not impressive compared to the combinations of wash solutions. Interestingly, there was no significant difference in bacterial reduction between 5 and 20 min of agitation. Results suggest that 5 min of agitation might be useful to inhibit both L. monocytogenes (Figure 2a) and Salmonella spp. (Figure 2b). In a similar study, 100\% lemon juice reduced populations of $Y$. enterocolitica by $7.2 \log \mathrm{CFU} / \mathrm{g}$ in $15 \mathrm{~min}$ [11]. It is also reported that acetic acid (2\%) and lemon juice (98\%) exhibited $1.7 \log \mathrm{CFU} / \mathrm{g}$ and $5 \mathrm{log} \mathrm{CFU} / \mathrm{g}$ of bacterial reduction against $L$. monocytogenes and E. coli 0157:H7, respectively [28]. Similarly, it is found that the combination of EOs and acetic acid reduced the $E$. coli 0157:H7 by more than $3 \log$ CFU/g and Salmonella by $2.5 \log$ CFU/g on spinach leaves without affecting leaves color and texture [29]. However, use of white vinegar at high concentration for 10 min resulted in sour taste and it caused an acceptability problem [30].
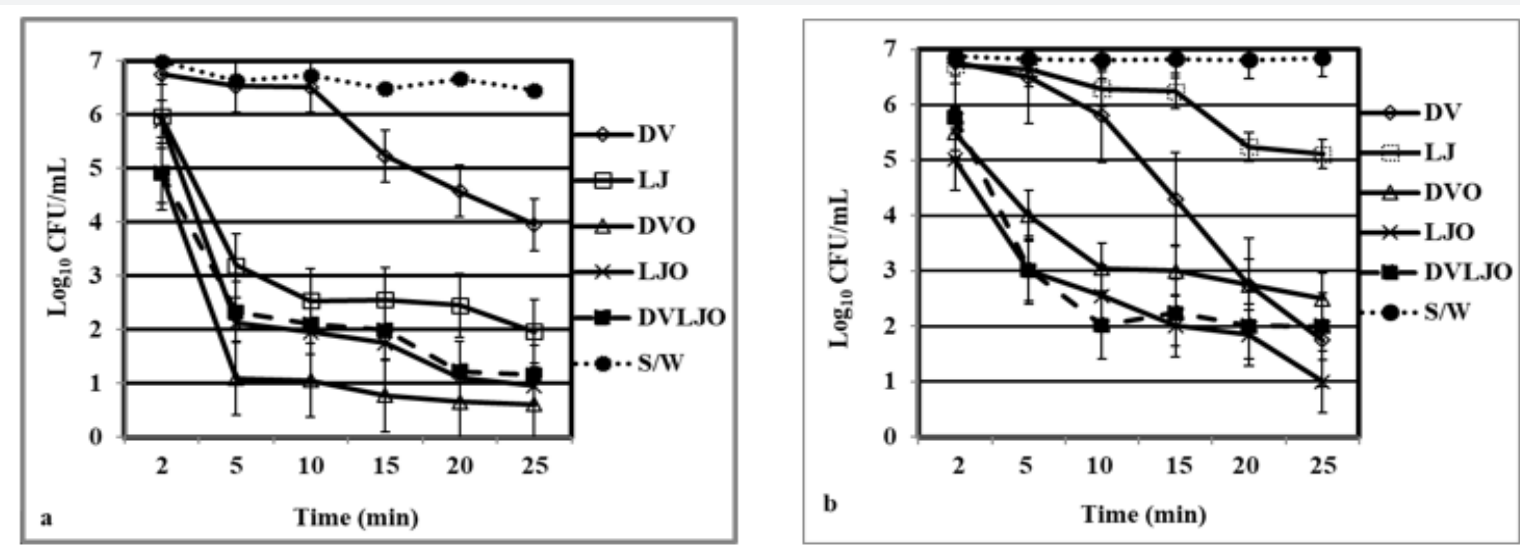

Figure 2: Efficacy of antimicrobial solutions at different times against a) L. monocytogenes and b) S. Typhimurium. DV: $0.5 \%$ white distilled vinegar, O: $0.1 \%$ origanum oil, LJ: $25 \%$ crude lemon juice, DVO: $0.5 \%$ white distilled vinegar $+0.1 \%$ origanum oil, LJO: $25 \%$ crude lemon juice $+0.1 \%$ origanum oil, DVLJO: $0.5 \%$ white distilled vinegar $+25 \%$ crude lemon juice $+0.1 \%$ origanum oil, S/W: sterile water (control).emon juice $+0.1 \%$ origanum oil, DVLJO: $0.5 \%$ white distilled vinegar $+25 \%$ crude lemon juice $+0.1 \%$ origanum oil, S/W: sterile water (control).

Table 3: Mean values of optical density (OD) at $600 \mathrm{~nm}$ of various concentrations of antimicrobial solutions against L. monocytogenes.

\begin{tabular}{|c|c|c|c|c|c|c|c|}
\hline \multicolumn{9}{|c|}{ OD at selected concentrations (\%) } \\
\hline Treatment & $\mathbf{1}$ & $\mathbf{0 . 5}$ & $\mathbf{0 . 2 5}$ & $\mathbf{0 . 1 2 5}$ & $\mathbf{0 . 0 6}$ & $\mathbf{0 . 0 3}$ & $\mathbf{0 . 0 1 5}$ \\
\hline DV $^{2)}$ & $0.07^{\mathrm{c} 3)}$ & $0.05^{\mathrm{c}}$ & $0.05^{\mathrm{c}}$ & $0.06^{\mathrm{c}}$ & $0.35^{\mathrm{b}}$ & $0.54^{\mathrm{ab}}$ & $0.58^{\mathrm{a}}$ \\
\hline LJ & $0.42^{\mathrm{b}}$ & $0.24 \mathrm{~b}^{\text {cd }}$ & $0.15^{\mathrm{d}}$ & $0.10^{\mathrm{d}}$ & $0.21^{\text {cd }}$ & $0.35^{\mathrm{bc}}$ & $0.68^{\mathrm{a}}$ \\
\hline DVO & $0.07^{\mathrm{b}}$ & $0.05^{\mathrm{b}}$ & $0.05^{\mathrm{b}}$ & $0.06^{\mathrm{b}}$ & $0.40^{\mathrm{a}}$ & $0.50^{\mathrm{a}}$ & $0.54^{\mathrm{a}}$ \\
\hline LJO & $0.62^{\mathrm{a}}$ & $0.3 \mathrm{~b}^{\mathrm{c}}$ & $0.18^{\mathrm{c}}$ & $0.11^{\mathrm{c}}$ & $0.12^{\mathrm{c}}$ & $0.38^{\mathrm{b}}$ & $0.47^{\mathrm{ab}}$ \\
\hline DVJJO & $0.38^{\mathrm{ab}}$ & $0.16^{\text {cd }}$ & $0.10^{\mathrm{d}}$ & $0.08^{\mathrm{d}}$ & $0.22^{\mathrm{bcd}}$ & $0.35^{\mathrm{abc}}$ & $0.43^{\mathrm{a}}$ \\
\hline SE $^{11}$ & 0.07 & 0.07 & 0.07 & 0.07 & 0.07 & 0.07 & 0.07 \\
\hline
\end{tabular}

1)SE: Standard error.

${ }^{2)} \mathrm{DV}$ : $0.5 \%$ white distilled vinegar; O: $0.1 \%$ origanum oil, LJ: $25 \%$ crude lemon juice; DVO: $0.5 \%$ white distilled vinegar + 0.1\% origanum oil; LJO: $25 \%$ crude lemon juice $+0.1 \%$ origanum oil; DVLJO: $0.5 \%$ white distilled vinegar $+25 \%$ crude lemon juice $+0.1 \%$ origanum oil.

3)'abcd' Treatment mean across the row and within the column do not follow by common superscript differ $(p<0.05)$.

Table 4: Mean values of optical density (OD) at $600 \mathrm{~nm}$ of various concentrations of antimicrobials solutions against S. Typhimurium.

\begin{tabular}{|c|c|c|c|c|c|c|c|}
\hline \multicolumn{8}{|c|}{ OD at selected concentrations (\%) } \\
\hline Treatment & 1 & 0.5 & 0.25 & 0.125 & 0.06 & 0.03 & 0.015 \\
\hline $\mathrm{DV}^{2)}$ & $0.06^{\mathrm{c3})}$ & $0.05^{c}$ & $0.06^{\mathrm{c}}$ & $0.06^{c}$ & $0.54^{\mathrm{b}}$ & $1.09^{\mathrm{a}}$ & $1.09^{\mathrm{a}}$ \\
\hline LJ & $0.34^{\mathrm{b}}$ & $0.23^{\mathrm{c}}$ & $0.15^{c}$ & $0.11^{\mathrm{c}}$ & $0.36^{\mathrm{b}}$ & $0.46^{\mathrm{b}}$ & $1.04^{\mathrm{a}}$ \\
\hline DVO & $0.07^{\mathrm{c}}$ & $0.05^{\mathrm{c}}$ & $0.06^{\mathrm{c}}$ & $0.06^{\mathrm{c}}$ & $0.44^{\mathrm{b}}$ & $1.04^{\mathrm{a}}$ & $1.02^{\mathrm{a}}$ \\
\hline LJO & $0.55^{\mathrm{b}}$ & $0.35^{\mathrm{c}}$ & $0.20^{\mathrm{de}}$ & $0.13^{\mathrm{e}}$ & $0.32^{\text {cd }}$ & $0.48^{\mathrm{b}}$ & $0.94^{\mathrm{a}}$ \\
\hline DVLJO & $0.32^{\mathrm{c}}$ & $0.15^{\mathrm{d}}$ & $0.14^{\mathrm{d}}$ & $0.09^{\mathrm{d}}$ & $0.31^{\mathrm{c}}$ & $0.63^{\mathrm{b}}$ & $0.83^{\mathrm{a}}$ \\
\hline $\mathrm{SE}^{1)}$ & 0.04 & 0.04 & 0.04 & 0.04 & 0.04 & 0.04 & 0.04 \\
\hline
\end{tabular}

1)SE: Standard error.

${ }^{2}$ DV: $0.5 \%$ white distilled vinegar; O: $0.1 \%$ origanum oil, LJ: $25 \%$ crude lemon juice; DVO: $0.5 \%$ white distilled vinegar + $0.1 \%$ origanum oil; LJO: $50 \%$ crude lemon juice $+0.1 \%$ origanum oil; DVLJO: $0.5 \%$ white distilled vinegar $+25 \%$ crude lemon juice $+0.1 \%$ origanum oil.

3)'abcde' Treatment mean across the row and within the column do not follow by common superscript differ $(p<0.05)$. 


\section{Conclusion}

This study showed that formulated wash prepared from natural ingredients was effective to inhibit foodborne pathogenic bacteria, and combined treatments were preferred to be used as potential natural wash rather than single use of natural ingredient. Our results illustrate that a combination of vinegar, lemon and essential oil could be an alternative wash to avoid harmful chemical wash as well as to prevent illness caused from consumption of raw or minimally processed fresh produce. However, it is suggested that further validation and application on the surface of fresh produce with various morphological properties by green wash using natural ingredients, and how it affects other properties such as nutritional and sensory properties are recommended.

\section{Acknowledgment}

This research is supported by USDA NIFA Evans-Allen research program and George Washington Carver Agricultural Experiment Station at Tuskegee University.

\section{Conflicts of interest}

The authors declare that no conflict of interest.

\section{References}

1. Yang Y, Meier F, Ann Lo J, Yuan W, Lee Pei Sze V, et al. (2013) Overview of recent events in the microbiological safety of sprouts and new intervention technologies. Compr Rev Food Sci Food Saf 12(3): 265-280.

2. Olaimat AN, Holley RA (2012) Factors influencing the microbial safety of fresh produce: A review. Food Microbiol 32(1): 1-19.

3. Ouedrhiri W, Balouiri M, Bouhdid S, Moja S, Chahdi FO, et al. (2016) Mixture design of Origanum compactum, Origanum majorana and Thymus serpyllum essential oils: Optimization of their antibacterial effect. Ind Crops Prod 89: 1-9.

4. Akbas MY, Ölmez H. (2007) Inactivation of Escherichia coli and Listeria monocytogenes on iceberg lettuce by dip wash treatments with organic acids. Lett Appl Microbiol 44(6): 619-624.

5. Raybaudi-Massilia RM, Mosqueda-Melgar J, Soliva-Fortuny R, MartínBelloso 0 (2009) Control of pathogenic and spoilage microorganisms in fresh-cut fruits and fruit juices by traditional and alternative natural antimicrobials. Compr Rev Food Sci Food Saf 8(3): 157-180.

6. Dutra TV, Castro JC, Menezes JL, Ramos TR, do Prado IN, et al. (2019) Bioactivity of oregano (Origanum vulgare) essential oil against Alicyclobacillus spp. Ind Crops Prod 129: 345-349.

7. Bhavaniramya S, Vishnupriya S, Al-Aboody MS, Vijayakumar R, Baskaran D (2019) Role of essential oils in food safety: Antimicrobial and antioxidant applications. Grain Oil Sci Technol 2: 49-55.

8. Yossa N, Patel J, Miller P, Lo YM (2010) Antimicrobial activity of essential oils against Escherichia coli 0157:H7 in organic soil. Food Control 21(11): 1458-1465.

9. Ho CW, Lazim AM, Fazry S, Zaki UKHH, Lim SJ (2017) Varieties, production, composition and health benefits of vinegars: A review. Food Chem 221: 1621-1630.

10. Yang J, Lee D, Afaisen S, Gadi R (2013) Inactivation by lemon juice of Escherichia coli 0157: H7, Salmonella Enteritidis, and Listeria monocytogenes in beef marinating for the ethnic food kelaguen. Int J Food Microbiol 160(3): 353-359.

11. Sengun IY, Karapinar M (2004) Effectiveness of lemon juice, vinegar and their mixture in the elimination of Salmonella Typhimurium on carrots (Daucus carota L.). Int J Food Microbiol 96(3): 301-305.
12. Lingham T, Besong S, Ozbay G (2012) Antimicrobial Activity of Vinegar on Bacterial Species Isolated from Retail and Local Channel Catfish (Ictalurus punctatus). J Food Process Technol 01(S11): 1-5.

13. Stefanakis MK, Touloupakis E, Anastasopoulos E, Ghanotakis D, Katerinopoulos HE, et al. (2013). Antibacterial activity of essential oils from plants of the genus Origanum. Food Control 34(2): 539-546.

14. Huang K, Nitin N (2019) Antimicrobial particle-based novel sanitizer for enhanced decontamination of fresh produce. Appl Environ Microbiol 85(8): e02599-e02618.

15. Sun X, Baldwin E, Bai J (2019) Applications of gaseous chlorine dioxide on postharvest handling and storage of fruits and vegetables - A review. Food Control 95: 18-26.

16. Fu TJ, Li Y, Awad D. Zhou TY, Liu L (2018) Factors affecting the performance and monitoring of a chlorine wash in preventing Escherichia coli 0157:H7 cross-contamination during postharvest washing of cut lettuce. Food Control 94: 212-221.

17. Kim JG, Yousef AE, Dave S (1999) Application of ozone for enhancing the microbiological safety and quality of foods: A review. J Food Prot 62(9): 1071-1087.

18. Richardson SD, Plewa MJ, Wagner ED, Schoeny R, DeMarini DM (2007) Occurrence, genotoxicity, and carcinogenicity of regulated and emerging disinfection by-products in drinking water: A review and roadmap for research. Mutat Res 636(1-3): 178-242.

19. Lee G, K im Y, Kim H, Beuchat LR, Ryu JH (2018) Antimicrobial activities of gaseous essential oils against Listeria monocytogenes on a laboratory medium and radish sprouts. Int J Food Microbiol 265: 49-54.

20. Gill AO, Holley RA (2006) Disruption of Escherichia coli, Listeria monocytogenes and Lactobacillus sakei cellular membranes by plant oil aromatics. Int J Food Microbiol 108(1): 1-9.

21. De Azeredo GA, De Figueiredo RCBQ, De Souza EL, Stamford TLM (2012) Changes in Listeria monocytogenes induced by Origanum vulgare $L$. and Rosmarinus officinalis $L$. Essential oils alone and combined at subinhibitory amounts. J Food Saf 32(2): 226-235.

22. Souza EL, Stamford TLM, Lima EO, Trajano VN (2007) Effectiveness of Origanum vulgare $L$. essential oil to inhibit the growth of food spoiling yeasts. Food Control 8(5): 409-413.

23. Murbach Teles Andrade BF, Nunes Barbosa L, Da Silva Probst I, Fernandes Júnior A (2014) Antimicrobial activity of essential oils. J Essent Oil Res 26(1): $34-40$.

24. Yang H, Kendall PA, Medeiros L, Sofos JN (2009) Inactivation of Listeria monocytogenes, Escherichia coli 0157:H7, and Salmonella Typhimurium with compounds available in households. J Food Prot 72(6): 1201-1218.

25. Ibrahim L, Karaky M, Ayoub P, El Ajouz N, Ibrahim S (2012) Chemical composition and antimicrobial activities of essential oil and its components from Lebanese Origanum syriacum L.J Essent Oil Res 24(4): 339-345.

26. Romeo FV, Luca S De, Piscopo A, Poiana M (2008) Antimicrobial Effect of Some Essential Oils. J Essent Oil Res 20(4): 375-379.

27. Cunningham E, O'Byrne C, Oliver JD (2009) Effect of weak acids on Listeria monocytogenes survival: Evidence for a viable but nonculturable state in response to low pH. Food Control 20(12): 1141-1144.

28. Ssemanda JN, Joosten H, Bagabe MC, Zwietering MH, Reij MW (2018) Reduction of microbial counts during kitchen scale washing and sanitization of salad vegetables. Food Control 85: 495-503.

29. Vijayakumar C, Wolf-Hall CE (2002) Evaluation of household sanitizers for reducing levels of Escherichia coli on iceberg lettuce. J Food Prot 65(10): 1646-1650.

30. Yossa N, Patel J, Millner P, Lo YM (2012) Essential oils reduce Escherichia coli 0157:H7 and Salmonella on spinach leaves. J Food Prot 75(3): 488496. 